\title{
Fabrication of Arrays of Metal and Metal Oxide Nanotubes by Shadow Evaporation
}

\section{Citation}

Dickey, Michael D., Emily A. Weiss, Elizabeth J. Smythe, Ryan C. Chiechi, Federico Capasso, and George M. Whitesides. 2008. "Fabrication of Arrays of Metal and Metal Oxide Nanotubes by Shadow Evaporation." ACS Nano 2 (4): 800-808. https://doi.org/10.1021/nn800036r.

\section{Permanent link}

http://nrs.harvard.edu/urn-3:HUL.InstRepos:41371608

\section{Terms of Use}

This article was downloaded from Harvard University's DASH repository, and is made available under the terms and conditions applicable to Open Access Policy Articles, as set forth at http:// nrs.harvard.edu/urn-3:HUL.InstRepos:dash.current.terms-of-use\#OAP

\section{Share Your Story}

The Harvard community has made this article openly available.

Please share how this access benefits you. Submit a story.

Accessibility 


\title{
Fabrication of Arrays of Metal and Metal Oxide Nanotubes by Shadow Evaporation
}

\author{
Michael D. Dickey, ${ }^{\dagger}$ Emily A. Weiss, ${ }^{\dagger}$ Elizabeth J. Smythe, ${ }^{\ddagger}$ Ryan C. Chiechi, ${ }^{\dagger}$ Federico Capasso, ${ }^{\ddagger}$ and \\ George M. Whitesides ${ }^{\text {,* }}$ \\ ${ }^{\dagger}$ Department of Chemistry and Chemical Biology, Harvard University, 12 0xford Street, Cambridge, Massachusetts 02138, and ${ }^{\ddagger}$ School of Engineering and Applied Sciences, \\ Harvard University, 29 0xford Street, Cambridge, Massachusetts 02138
}

ABSTRACT This paper describes a simple technique for fabricating uniform arrays of metal and metal oxide nanotubes with controlled heights and diameters. The technique involves depositing material onto an anodized aluminum oxide $(\mathrm{AAO})$ membrane template using a collimated electron beam evaporation source. The evaporating material enters the porous openings of the AAO membrane and deposits onto the walls of the pores. The membrane is tilted with respect to the column of evaporating material, so the shadows cast by the openings of the pores onto the inside walls of the pores define the geometry of the tubes. Rotation of the membrane during evaporation ensures uniform deposition inside the pores. After evaporation, dissolution of the AAO in base easily removes the template to yield an array of nanotubes connected by a thin backing of the same metal or metal oxide. The diameter of the pores dictates the diameter of the tubes, and the incident angle of evaporation determines the height of the tubes. Tubes up to $\sim 1.5 \mu \mathrm{m}$ in height and $20-200 \mathrm{~nm}$ in diameter were fabricated. This method is adaptable to any material that can be vapor-deposited, including indium - tin oxide (ITO), a conductive, transparent material that is useful for many opto-electronic applications. An array of gold nanotubes produced by this technique served as a substrate for surface-enhanced Raman spectroscopy: the Raman signal (per molecule) from a monolayer of benzenethiolate was a factor of $\sim 5 \times 10^{5}$ greater than that obtained using bulk liquid benzenethiol.

KEYWORDS: shadow evaporation - nanotube arrays · SERS (surface-enhanced Raman spectroscopy) · templated fabrication · ITO (indium - tin oxide) · AAO (anodized aluminum oxide) $\cdot$ nanofabrication · line-of-sight deposition

*Address correspondence to gwhitesides@gmwgroup.harvard.edu.

Received for review January 21, 2008 and accepted March 03, 2008.

Published online March 22, 2008. 10.1021/nn800036r CCC: $\$ 40.75$

๑) 2008 American Chemical Society

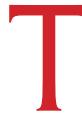

his paper describes a simple method for the fabrication of electrically continuous arrays of nanotubes of platinum, gold, and indiumdoped tin oxide (ITO) with controllable dimensions (height and outer diameter). The two steps in the procedure (Figure 1) are (i) electron-beam (e-beam) evaporation of the metal or metal oxide onto rotating, nanoporous anodized aluminum oxide (AAO) membranes (also used in the electrochemical template synthesis method developed by Martin ${ }^{1}$ ) oriented at an angle $(\alpha)$ of $0-90^{\circ}$ between the axis of rotation and the direction of evaporation (where $\alpha$ determines the height of the tubes) and (ii) dissolution of the membrane. The method presented here is more convenient than several other multistep procedures for fabricating arrays of nanotubes, ${ }^{2-5}$ enables better control over the dimensions of the nanotubes than do conventional ${ }^{6}$ sputtering methods, ${ }^{7-9}$ chemical vapor deposition, ${ }^{10}$ and sol-gel processing, ${ }^{11}$ and, unlike electrodeposition, ${ }^{1,12,13}$ is generally applicable to any material that can be vapordeposited. We demonstrate the utility of an array of Au nanotubes as a substrate for surface-enhanced Raman spectroscopy (SERS); without optimization, it provided an enhancement in the Raman signal of benzenethiolate that is competitive with that of substrates specifically designed for SERS applications. Furthermore, arrays of uniform nanotubes are potentially useful as electrodes for three-dimensional, nanostructured versions of devices such as solar cells, light-emitting diodes (LEDs), electrochromics, and batteries because they provide (i) a high ratio of surface area to volume for interfacial charge collection/ separation/ion transport across liquid/solid interfaces (i.e., for mass-transport-limited processes in general) and (ii) a template for depositing nanostructured films of small molecules or polymers that serve as optically and electronically active layers for these devices. ${ }^{14-17}$ There have been many efforts to fabricate nanostructures of ITO (the most popular transparent conducting oxide for use in such devices) ${ }_{i}^{18-26}$ this work is the first to produce uniform, freestanding ordered arrays of electrically connected ITO nanotubes with controllable dimensions.

\section{EXPERIMENTAL DESIGN}

Materials. We chose AAO membranes (Whatman Anodisc, $60 \mu \mathrm{m}$ thick, 200-nmdiameter pores) as templates because they are commercially available, dissolve easily in 
aqueous base, and have a high density $\left(\sim 10^{9}-10^{11} / \mathrm{cm}^{2}\right.$, depending on the pore size) of straight pores with monodisperse diameters (see the Results and Discussion section for quantitative analysis of the dispersity of the diameters). Alumina membranes with regular, cylindrical pores with diameters from $10 \mathrm{~nm}$ to micrometers can also be fabricated through established anodic processing techniques. . $^{37-31}$

In principle, the technique we describe is compatible with any material that can be vapor-deposited. To illustrate the method, we focused on three materials: gold, platinum, and ITO. Gold and platinum are useful as electrodes for analytical electrochemistry and for electrodeposition of conducting polymers, because these metals do not oxidize within the electrochemical window of common solvent/ electrolyte systems. ITO, unlike nanostructures of titania ${ }^{32-37}$ and metals, ${ }^{38-44}$ has both high electrical conductivity and transparency throughout much of the UV and visible regions of the spectrum. These properties make ITO useful in many commercial applications, including flat panel displays, LEDs, and solar cells. Although many of these applications would benefit from high-surface-area electrodes, few techniques exist for creating welldefined nanostructures of ITO.

Electron-Beam Evaporation. In contrast to other deposition techniques (e.g., sputtering), the evaporating material produced by an electron beam has a long mean free path due to the low pressure in the chamber $(<1$ $\times 10^{-6}$ Torr), and, thus, is approximately collimated after traveling from the source to the substrate $(\sim 30 \mathrm{~cm}$ total distance). The collimated beam is critical for this application because the shadows cast by the AAO membrane into its pores define the geometry of the tubes. There do exist specialized line-of-sight sputtering techniques that use ion beam sources ${ }^{45,46}$ or collimator screens, ${ }^{9}$ but these techniques are not commonly used because of their low rates of deposition and need for specialized equipment. Sputter redeposition has also been used to form arrays of nanotubes, but this multistep process requires ion beam sputtering plus the use of a delicate (300-nm-thick) AAO stencil mask. ${ }^{47}$ Electron-beam evaporation is appealing because it is a straightforward technique that is capable of depositing a wide range of high-purity materials with high rates of deposition (as much as several micrometers per minute ${ }^{9}$ ).

\section{RESULTS AND DISCUSSION}

Fabrication of the Arrays of Nanotubes. Figure 1 shows the general procedure for fabricating arrays of nano-
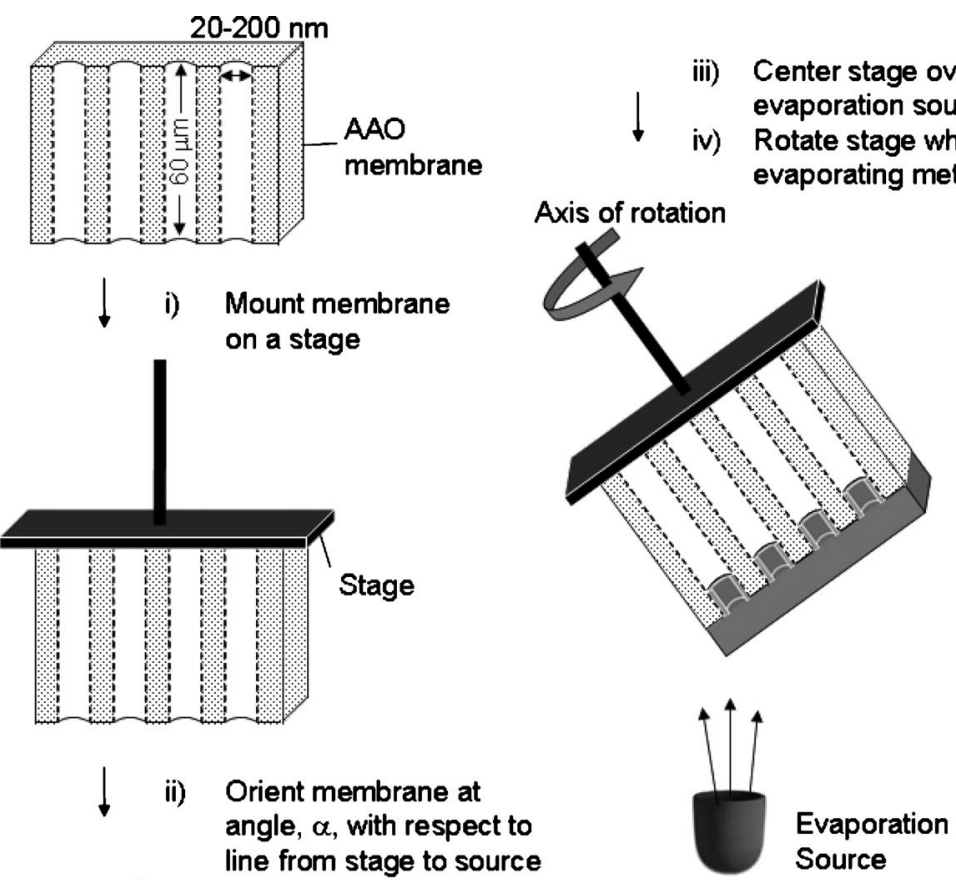

ii) Orient membrane at angle, $\alpha$, with respect to line from stage to source Source

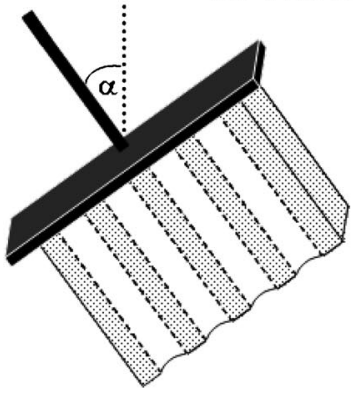

v) Remove from stage and dissolve membrane

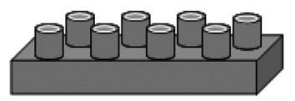

Figure 1. Procedure for the fabrication of nanotube arrays. We (i) mounted an anodized alumina (AAO) membrane onto a stage, (ii),(iii) centered the stage directly above the evaporation source at an incident angle $\alpha$ (defined as the angle between the axis of rotation and a line drawn from the source to the AAO membrane), and (iv) evaporated metal onto the membrane. The edges of the pores of the membrane cast shadows into the pores. (v) We immersed the coated membrane in $1 \mathrm{M} \mathrm{NaOH}$ for $1 \mathrm{~h}$ to completely tch the AAO membrane and yield an array of nanotubes connected by a continuous backing of the same material.

tubes. We mounted the AAO membranes flush against a rotating plate driven by a battery-powered DC motor ( $\sim 30 \mathrm{rpm}$ ) and e-beam evaporated $\sim 150 \mathrm{~nm}$ of metal (Au or Pt) or ITO onto the membranes. We fixed the evaporation incident angle ( $\alpha$ ) between 0 and $90^{\circ}$ to control the height of the nanotubes. We define $\alpha$ as the angle between the axis of rotation and a hypothetical line between the source and the AAO membrane. After deposition, we removed the AAO membrane from the rotating support. We annealed the ITO-AAO composite in an oven at $350^{\circ} \mathrm{C}$ for $1 \mathrm{~h}$ to convert the asdeposited, opaque ITO into a transparent and electrically conductive form. ${ }^{48}$ We performed no annealing steps on the deposited metal structures. The alumina dissolved when the AAO-metal (or ITO) composite was immersed in $1 \mathrm{M} \mathrm{NaOH}(\mathrm{aq})$ for $1 \mathrm{~h}$. This procedure yielded an array of nanotubes supported by an electrically continuous backing of the same material. The thickness of this backing layer depended on the angle 

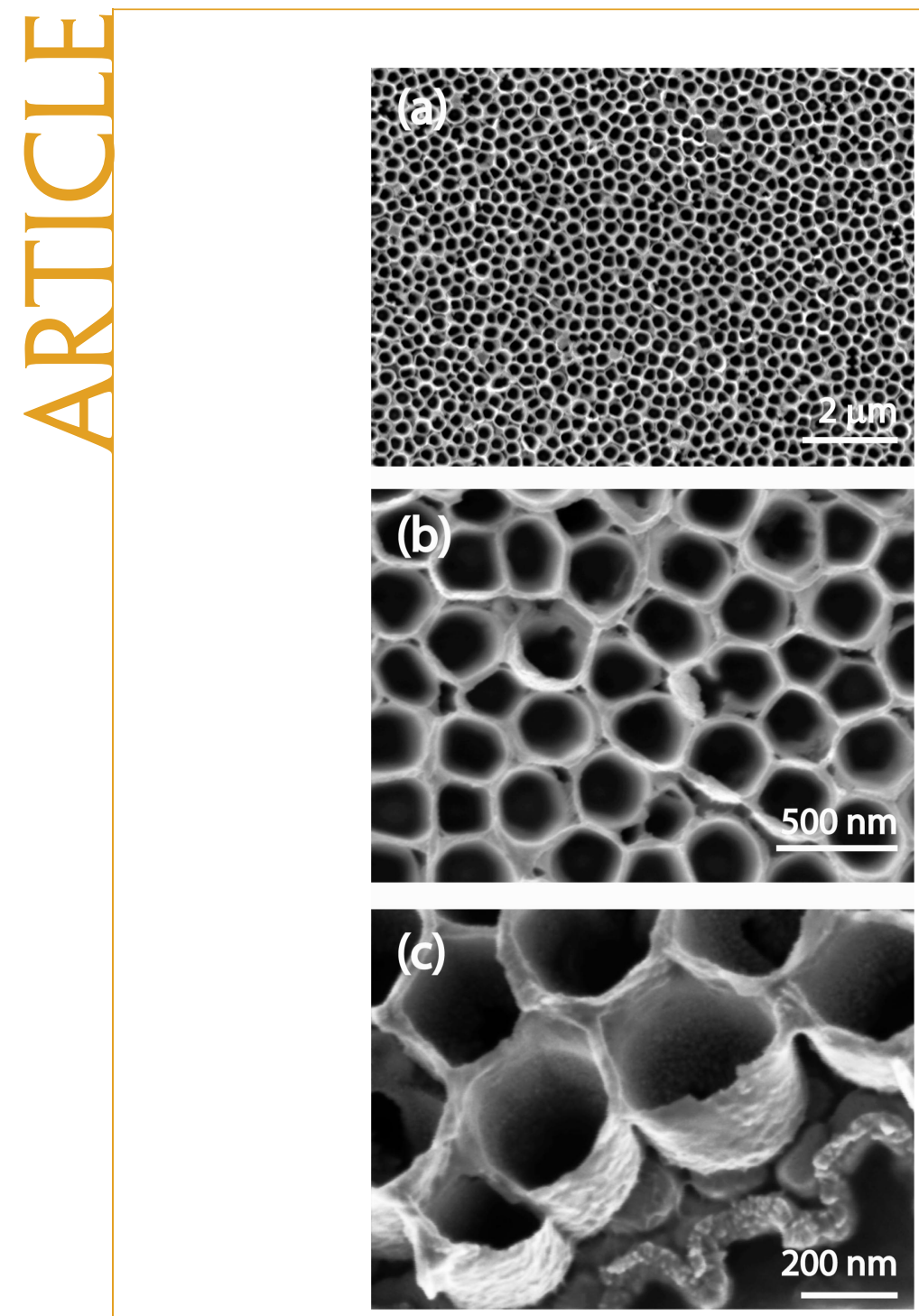

Figure 2. SEM images (with increasing magnification from (a) to (c)) of the Pt nanostructures prepared by evaporation at an incident angle $\alpha=45^{\circ}$. The walls of these tubes are $\sim 200 \mathrm{~nm}$ tall and $\sim 10 \mathrm{~nm}$ thick. The average diameter of the tubes (over an area of $100 \mu \mathrm{m}^{2}$ ) is $193 \pm 29 \mathrm{~nm}$.

of evaporation and the amount of deposited material, which was monitored by a quartz crystal microbalance.

Microscopic Characterization of the Nanotubes. Figure 2 shows three scanning electron microscopy (SEM) images, with increasing magnification from (a) to (c), of Pt nanotubes evaporated at $\alpha=45^{\circ}$, after the dissolution of the AAO membrane. The nanotubes are close-packed and are uniform in diameter, height $(\sim 200 \mathrm{~nm})$, and wall thickness. We estimate that the walls of the tubes are $\sim 10 \mathrm{~nm}$ thick, on the basis of the SEM images. The nanotubes in Figure 2a have an average inner diameter of $193 \pm 29 \mathrm{~nm}$. We determined this value by measuring the cross-sectional area of the opening in each tube from the top-down SEM image in Figure 2a using ImageJ image analysis software (see Supporting Information), calculating the inner diameter of each tube from its area, and analyzing the distribution of diameters over the area of the representative image $(9.3 \mu \mathrm{m} \times$ $11.3 \mu \mathrm{m}, \sim 1000$ tubes). The pristine AAO membrane

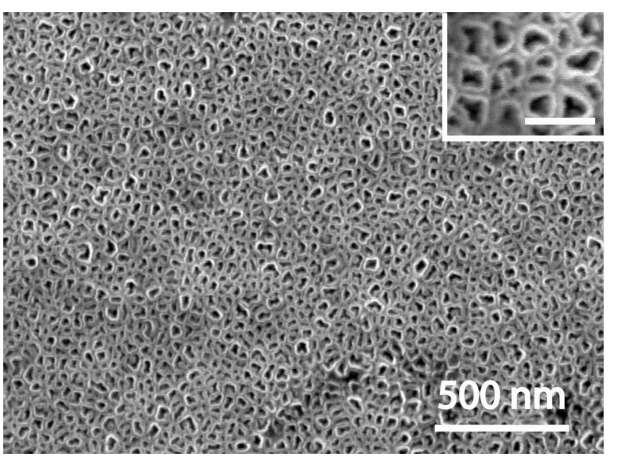

Figure 3. Top-down SEM image of ITO nanotubes prepared by evaporation at an incident angle $\alpha=45^{\circ}$ onto the branched side of an AAO membrane with nominal 20-nm pores. Inset: Close-up of tubes (scale bar, $100 \mathrm{~nm}$ ). All membranes have 200-nm-diameter cylindrical pores that span the thickness of the membrane. Each of these cylindrical pores branches into smaller pores on the "branched side" of the membrane; the average width of these pores is described by the advertised diameter for the membrane (here, $20 \mathrm{~nm})$.

had an average diameter of $215 \pm 26 \mathrm{~nm}$ over an identical area; our method, therefore, effectively replicated the pore diameters of the bare AAO membranes.

Scalability of the Technique. The accurate replication of the AAO pore structure implies that the array of tubes formed when the collimated, vaporized material penetrated the opening of the pores and deposited on the walls of the cylindrical pores. Based on this mechanism, the shadow evaporation technique should extend to pores with diameters smaller than $200 \mathrm{~nm}$. The AAO membranes are commercially available with pores as small as $20 \mathrm{~nm}$ in diameter (Whatman Anodisc), although these dimensions are defined by a web-like mesh of branched tubes rather than regular, cylindrical pores extending across the thickness of the membrane. Figure 3 is a top-down SEM image of a web-like array of ITO tubes formed by evaporating into the 20-nm pores of the AAO membrane at $\alpha=45^{\circ}$. We were able to form arrays of nanotubes that effectively replicated pores with 20-, 100-, and 200-nm diameters (see Supporting Information, Figure S1) and the 50-nm pores of a polycarbonate membrane (for which the pore density, $\sim 4 \times 10^{8} / \mathrm{cm}^{2}$, is much lower than that of an AAO membrane with similarly sized pores, $\sim 10^{10}-10^{11}$ / $\mathrm{cm}^{2}$ ). Regardless of the irregularity of the arrays formed with the "branched" side of the AAO membrane and the polycarbonate membrane, these experiments suggest that this evaporation technique is capable of replicating templates of almost arbitrarily small dimensions, shape, and composition (including AAO membranes with regular, cylindrical pores as small as $20 \mathrm{~nm}$, which are not commercially available but have been produced using established anodization methods). ${ }^{3,27-31}$

Control of the Heights of the Nanotubes. The heights of the structures depended on the angle of evaporation $(\alpha$, the angle between the axis of rotation and a line between the 
(a)

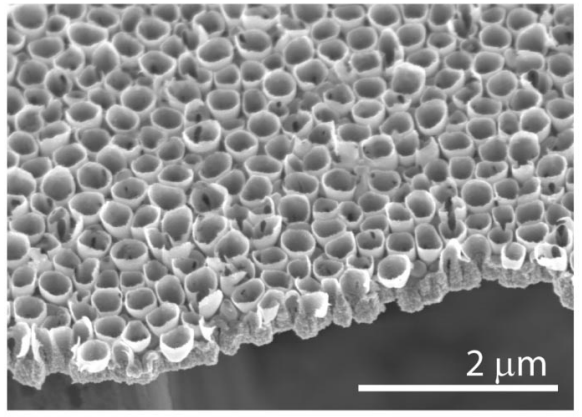

(b)

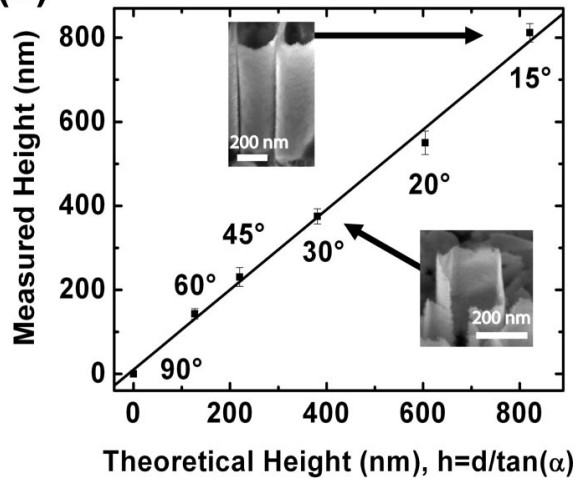

Figure 4. (a) SEM image of ITO nanostructures evaporated at $\alpha=45^{\circ}$. The heights and diameters of the tubes are $\sim 200$ $\mathrm{nm}$. (b) Plot of the measured height of the tubes versus the theoretical height of the tubes (calculated from eq 1). Each data point corresponds to an average of 10 height measurements (by inspection of cross-sectional SEM images) of tubes formed at an evaporation incident angle $\alpha$ as labeled on the plot. The error bars represent the standard deviation of these measurements (we assume a height of zero for $\alpha=$ $90^{\circ}$, see Figure $5 \mathrm{a}$ ). The measurements were made on tubes formed in membranes whose pores were $\sim 215 \mathrm{~nm}$ in diameter. The line represents the least-squares fit of the data forced through the origin (slope $=0.996, R^{2}=0.995$ ). Insets: Side-view SEM images of tubes formed at $\alpha=30^{\circ}$ and $\alpha=15^{\circ}$. The tubes were cleaved upon preparing the cross section of the sample.

source and the AAO membrane). Figure 4a shows structures formed by evaporating ITO at $\alpha=45^{\circ}$. Figure $4 \mathrm{~b}$ is a plot of the measured heights of the tubes versus the theoretical heights of the tubes for various angles of evaporation (as labeled on the data) onto membranes with nominal 200-nm-diameter pores (we omitted the data obtained at a glancing angle because the theoretical height approaches infinity). We measured the heights of the tubes using cross-sectional SEM images, which we acquired by mounting a cleaved membrane on a $45^{\circ} \mathrm{SEM}$ sample stub and rotating the stub within the SEM chamber such that the field of view was normal to the exposed, cleaved edge of the membrane. The data in Figure 4 represent the averages of 10 height measurements taken over three or four SEM images. The theoretical height of the tubes, $h$, is given by eq 1 ,

$$
h=\frac{d}{\tan (\alpha)}
$$
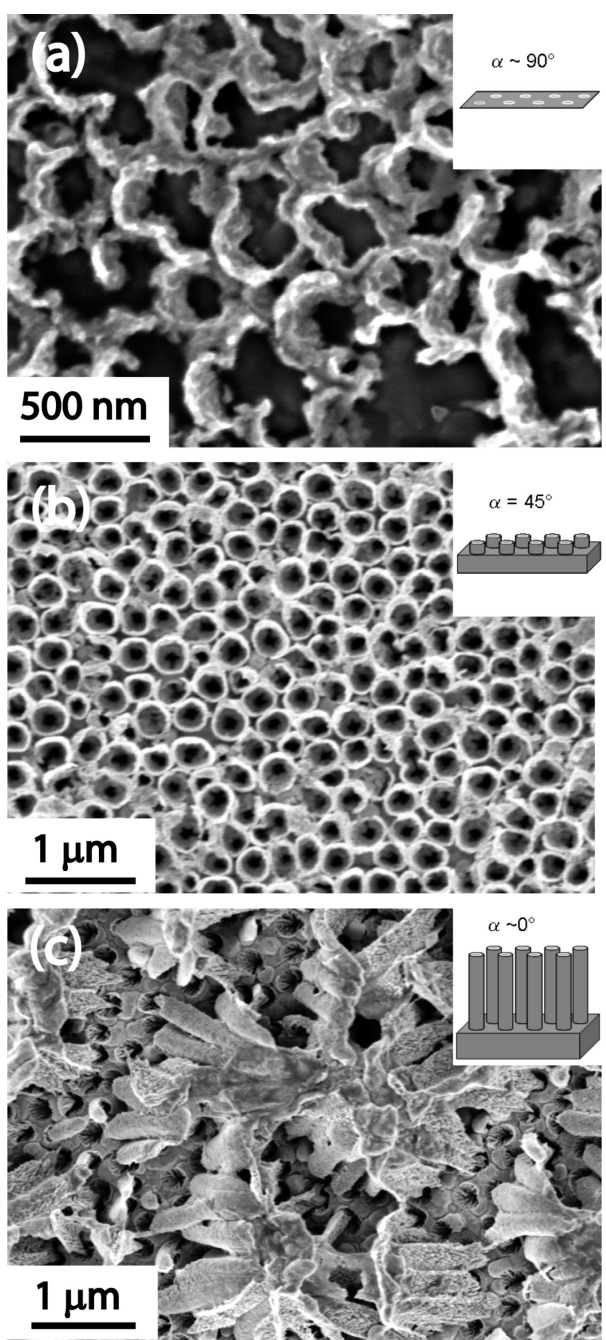

Figure 5. SEM images (top-view) of Au nanostructures formed at various incident angles $\alpha$ (insets: corresponding oblique-angle diagrams illustrating the dependence of the ideal geometry of the nanotube on $\alpha$ for a given amount of deposited material). (a) At $\alpha \approx 90^{\circ}$ (glancing), an array of small rings formed with a minimal backing layer. The collimated evaporation source resulted in negligible penetration into the pores and a thin backing layer. (b) At $\alpha \approx 45^{\circ}$, an array of short tubes formed. (c) At $\alpha \approx 0^{\circ}$ (normal), an array of tall, thin-walled tubes formed with a thick backing layer. The penetration was deep into the pores, but the walls of the tube were thin because the collimation of the vaporized material was parallel to the pores.

where $d$ is the diameter of the pores of the membrane (i.e., $215 \mathrm{~nm}$ ). A linear least-squares fit of the data (for which the $y$-intercept was fixed at 0) had a slope of 0.996 ( $R^{2}$ value of 0.995$)$; this result shows that the measured and theoretical heights in Figure $4 \mathrm{~b}$ are in close agreement.

Figure 5 shows micrographic evidence of the dependence of the height of Au tubes on the incident angle of evaporation for the two most extreme angles $\left(0^{\circ}, 90^{\circ}\right)$ and an intermediate angle $\left(45^{\circ}\right)$. At a glancing angle (Figure $5 \mathrm{a}, \alpha \approx 90^{\circ}$ ), the Au structures formed a loosely connected array of rings, because the collimated beam did not penetrate the pores, and only a small amount of 
deposited material adhered to the membrane. The diameter of the rings was $200 \mathrm{~nm}$ (the diameter of the pores of the AAO membrane). At $\alpha \approx 45^{\circ}$ (Figure $5 b$ ), the tubes formed structures with the height approximately the same as the diameter $(\sim 200 \mathrm{~nm})$, as predicted by eq 1 . At a normal angle (Figure $5 c, \alpha \approx 0^{\circ}$ ), the structures formed an array of long nanotubes $(\sim 1.5$ $\mu \mathrm{m}$ tall). These are the highest aspect ratio nanotubes formed using a one-step physical vapor deposition process on AAO membranes. The walls of the tubes, however, were thin since the collimated beam projected parallel to the walls of the pores. Many of the tubes in Figure $5 c$ therefore mechanically separated from the substrate or collapsed during sample preparation. In an attempt to minimize collapse of the tubes, we rinsed the samples with methanol-a low surface tension liquid-after rinsing off the $\mathrm{NaOH}$ solution with water and prior to drying; this additional rinsing step did not improve the structural stability of the tubes. We observed that the tubes were susceptible to this type of capillary-induced collapse at values of $\alpha<20^{\circ}$ (aspect ratios $>3: 1$ ).

As is characteristic of evaporated Au films, ${ }^{49}$ the surfaces of the walls of the Au tubes were grainier than those of the Pt tubes (Figure 2). After the Au was annealed at $200{ }^{\circ} \mathrm{C}$ for $2 \mathrm{~h}$, the walls of the tubes became visually smoother in the SEM, but the tubes deformed from their original dimensions.

Electrical and Optical Properties of the Arrays of ITO Nanotubes. The arrays of ITO nanotubes had a continuous, highly conductive backing of ITO. We measured the sheet resistance using a four-point probe (Creative Design Engineering, ResMap 178) with $1 \mathrm{~mm}$ spacing between the tips. The ITO nanotube film (Figure 3, 1 $\mathrm{cm}^{2}$ ) had a sheet resistance of $259.4 \pm 32.1 \Omega / \mathrm{sq}$; a film of ITO evaporated onto a featureless glass slide $(\sim 40$ $\mathrm{cm}^{2}$ ) had a sheet resistance of $108.6 \pm 8.9 \Omega / \mathrm{sq}$. The difference between these two values may be due to differences in the size of the sample (the ITO more nearly approximates an infinite plane, which is assumed to be present in the measurement of sheet resistance ${ }^{50}$ ). As has been observed previously for ITO formed by e-beam evaporation, ${ }^{51}$ both of the e-beam evaporated films were approximately a factor of 10 less conductive than commercially available ITO (Delta Technologies, CB-50IN; advertised sheet resistance $=5-15 \Omega / \mathrm{sq}$ ) formed by sputtering (7.8 $\pm 0.1 \Omega / \mathrm{sq})$.

Ultraviolet-visible absorption measurements (using a Hewlett-Packard 8453 UV-vis spectrometer) showed that the absorbance spectra $(300-1100 \mathrm{~nm})$ of the nanostructured films of ITO were indistinguishable from the spectrum of a featureless thin film of evaporated ITO on a glass slide: in both cases, the average transmission was $80 \%$ between 400 and $1100 \mathrm{~nm}$. We prepared samples for absorption measurements by gluing the ITO-coated AAO membrane onto a glass slide using Norland Optical Adhesive (NOA 65, transparent at wavelengths $>300 \mathrm{~nm}$ ) and dissolving the AAO. Scanning electron microscope images of the ITO surface confirmed that the nanotubes were present and that the glue did not penetrate the pores.

Application of Arrays of Metal Nanotubes as Substrates for Surface-Enhanced Raman Spectroscopy. Surface-enhanced Raman spectroscopy (SERS) is a technique for the detection of small concentrations (potentially single molecules) ${ }^{52,53}$ of analyte that are in close proximity of the intense electric fields generated by nanostructured metallic substrates upon photoexcitation at the wavelength of the metal's surface plasmon. The resulting signal enhancement is quantified by the enhancement factor, which is the ratio of the SERS signal (per molecule) to the signal (per molecule) of the bulk liquid. The total signal is maximized by maximizing the enhancement factor (a "per molecule" factor) and maximizing the number of molecules interrogated within the spot of the beam. An ideal SERS substrate would therefore have a high density of SERS-active sites. In addition, it is desirable to have a substrate that is simple and inexpensive to fabricate, significantly larger than the spot size of the interrogating optical beam, and uniform such that the signal across the entire substrate does not vary. We hypothesized that arrays of nanotubes would be wellsuited as SERS substrates because they have a dense number of nanostructures. Thus, we sought to study the SERS characteristics of arrays of Au nanotubes. Although there are many approaches for fabricating SERS substrates (e.g., embossing, colloidal lithography, e-beam lithography, electrochemical surface roughening), ${ }^{54-59}$ the fabrication method presented here is advantageous because it provides a simple route to producing dense arrays of uniform, thin-walled $(\sim 10$ $\mathrm{nm}$ thick), metallic nanostructures over a large area $\left(\mathrm{cm}^{2}\right)$.

We acquired Raman spectra of a self-assembled monolayer ${ }^{56,60,61}$ (SAM) of benzenethiolate on an array of Au nanotubes (Au-BT, in which the nanotubes were $\sim 200 \mathrm{~nm}$ high and $\sim 200 \mathrm{~nm}$ in diameter) and compared the intensity of the Raman signal to that of a spectrum acquired using liquid-phase, neat benzenethiol (BT). For information on the preparation of these samples, see the Supporting Information.

We obtained Raman spectra using a confocal microprobe Raman system (LabRam 1) based on an Olympus BX40 microscope. Light from a HeNe laser $(\lambda=$ $632.8 \mathrm{~nm}$ with $3 \mathrm{~mW}$ of power at the focal plane of the sample) was focused onto the sample using a $50 \times$ Olympus MPlan objective (working distance $0.38 \mathrm{~mm}$, $\mathrm{NA}=0.75$ ). The confocal microscope pinhole had a diameter of $500 \mu \mathrm{m}$. The microscope objective collected the light scattered off the sample and directed it to a CCD camera with an entrance slit width of $250 \mu \mathrm{m}$. We calibrated the detector using the $520 \mathrm{~cm}^{-1}$ peak of a Si/ $\mathrm{SiO}_{2}$ wafer. For each measurement, we averaged 10 spectra, where each spectrum was integrated for $10 \mathrm{~s}$. 
Figure 6 shows the SERS spectrum of the Au-BT sample (an average of spectra acquired over seven different locations). The characteristic Raman-active vibrational modes of benzenethiolate have energies 998 and 1021 (in-plane bending modes of the phenyl ring), 1072 ( $\mathrm{C}-\mathrm{H}$ in-plane bending and stretching mode), and $1571 \mathrm{~cm}^{-1}$ (C-C stretching mode). ${ }^{62}$

Equation 2 defines the surface enhancement factor, EF.

$$
\mathrm{EF}=\left(\frac{I_{\text {surf }}}{I_{\text {std }}}\right)\left(\frac{N_{\text {std }}}{N_{\text {surf }}}\right)
$$

In eq $2, I_{\text {surf }}$ is the integrated intensity of a surfaceenhanced Raman peak for the Au-BT, $I_{\text {std }}$ is the integrated intensity of the same Raman peak for the BT standard, $N_{\text {surf }}$ is the number of BT molecules that contribute to the SERS signal on the Au-BT sample, and $N_{\text {std }}$ is the number of molecules that contribute to the Raman signal in the scattering volume of the BT standard.

We calculated EF for the peaks at 1072 and 1571 $\mathrm{cm}^{-1}$ by following the rigorous calculation method outlined by Le Ru et al. ${ }^{63}$ (further details in the Supporting Information). We have chosen this method because it explicitly defines the input parameters used to calculate EF; there are no "black-box" elements in this calculation. Equations $3 \mathrm{a}$ and $3 \mathrm{~b}$ define $N_{\text {std }}$ and $N_{\text {surf }}$.

$$
\begin{gathered}
N_{\text {std }} / A_{\text {eff }}=C_{\text {std }} H_{\text {eff }} \\
N_{\text {surf }} / A_{\text {eff }}=\mu_{\text {surf }} F_{\text {surf }}
\end{gathered}
$$

In eq $3 a, C_{\text {std }}$ (molecules $/ \mathrm{cm}^{3}$ ) is the concentration of BT in the standard and $H_{\text {eff }}(\mathrm{cm})$ is the effective height of the scattering volume of the standard. The parameter $A_{\text {eff }}\left(\mathrm{cm}^{2}\right)$ is the effective cross-sectional area of the scattering volume (for the standard) or the scattering area for the sample. The value of $A_{\text {eff }}$ is the effective spot size of the microscope and is, therefore, the same for the standard and the sample. ${ }^{63}$ The product of $H_{\text {eff }}$ and $A_{\text {eff }}$ is $V_{\text {eff }}$, the effective scattering volume (of the standard), within which the Raman signal is uniform and equal to the signal originating from the center of the focal plane. The effective volume accounts for the threedimensional spatial distributions of both the intensity of the excitation beam and the efficiency with which the scattered light is collected; these distributions are characteristic of the excitation source and the optics of the microscope. Using established methods, ${ }^{58}$ we empirically determined (see Supporting Information) the value of $H_{\text {eff }}$ such that, within the volume $V_{\text {eff }}$ each molecule contributes equally to the Raman signal—that is, the "per volume Raman signal" is uniform. In eq $3 \mathrm{~b}, \mu_{\text {surf }}$ (molecules $/ \mathrm{cm}^{2}$ ) is the molecular packing density of benzenthiolate on the Au substrate and $F_{\text {surf }}$ is the fraction of the surface area of the substrate that contributes to the SERS.

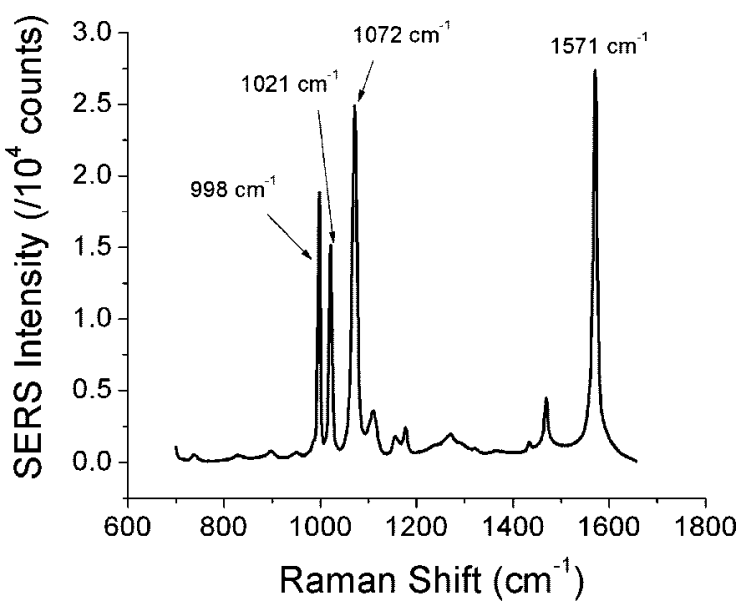

Figure 6. Raman spectrum of a monolayer of benzenethiolate on an array of Au nanotubes (200 nm diameter, 200 $\mathrm{nm}$ height, $\alpha=45^{\circ}$ ) The peaks occur at 998 and 1021 (inplane bending modes of the phenyl ring), $1072(\mathrm{C}-\mathrm{H}$ in-plane bending and stretching mode), and $1571 \mathrm{~cm}^{-1}(\mathrm{C}-\mathrm{C}$ stretching mode) The enhancement factors for the 1072 and 1571 $\mathrm{cm}^{-1}$ peaks are $4 \times 10^{5}$ and $5 \times 10^{5}$, respectively.

The ratio $N_{\text {std }} / A_{\text {eff }}$ is the product of the concentration of BT molecules $\left(C_{\text {std }}=5.9 \times 10^{21}\right.$ molecules/ $\mathrm{cm}^{3}$ ) and the effective height of the scattering volume $\left(H_{\text {eff }}=19 \times 10^{-4} \mathrm{~cm}\right)$ of the standard: $N_{\text {std }} / A_{\text {eff }}=1.1 \times$ $10^{19} \mathrm{~cm}^{-2}$.

To determine the ratio $N_{\text {surf }} / A_{\text {eff }}$ (eq $3 b$ ), we assumed that only the molecules at the rims of the nanotubes contributed significantly to the surface-enhanced Raman signal-that is, we considered the "enhancing" surface to be composed of an array of close-packed $\left(\sim 2.9 \times 10^{9}\right.$ tubes $\left./ \mathrm{cm}^{2}\right)$ rings with outer diameter 200 $\mathrm{nm}$ and inner diameter $190 \mathrm{~nm}$. The area of this surface is $\sim 6.3 \times 10^{-11} \mathrm{~cm}^{2}$ per tube. We believe this assumption is reasonable because the large majority of the Raman enhancement arises from large electric fields that are generated at the edges of nanoscale features. This assumption was justified further since the Raman signal from a monolayer of benzenethiolate on a smooth gold film (i.e., one without any nanoscale edges and a root-mean-square roughness of $\sim 1 \mathrm{~nm}$ over $25 \mu \mathrm{m}^{2}$ ) was undetectable using the same experimental setup as was used to collect the spectra of BT and Au-BT. The fractional surface coverage $F_{\text {surf }}$ in eq $3 \mathrm{~b}$ is the product of the area per nanotube and the number of nanotubes per unit area $\left(F_{\text {surf }}=0.18\right) .{ }^{64}$ For $\mu_{\text {surf }}$, we used the largest reported ${ }^{65}$ packing density of benzenethiolate on Au $\left(6.8 \times 10^{14}\right.$ molecules $/ \mathrm{cm}^{2}$; lower packing densities would only increase our calculated value of $\mathrm{EF}$ ). The product of $\mu_{\text {surf }}$ and $F_{\text {surf }}$ is $N_{\text {surf }} / A_{\text {eff }}=1.2 \times 10^{14} \mathrm{~cm}^{-2}$ (eq 3b).

Finally, we calculated EF using eq 2. We note that, since $A_{\text {eff }}$ is the same for both the sample and the standard, then $\left(N_{\text {surf }} / A_{\text {eff }}\right) /\left(N_{\text {std }} / A_{\text {eff }}\right)=N_{\text {surf }} / N_{\text {std }}$. The enhancement factor, $\mathrm{EF}$, is $5.3 \times 10^{5}$ at $1072 \mathrm{~cm}^{-1}$ and $3.6 \times 10^{5}$ at $1571 \mathrm{~cm}^{-1}$. The SERS literature has a wide range of reported EFs $\left(10^{4}-10^{14}\right)$, but a recent review 
on EF calculations suggests that "average SERS EFs" - in which "average" refers to the SERS signal averaged over the entire area of interrogation, not isolated "hot spots" on the surface- should be $\sim 10^{4}-10^{6} .{ }^{63}$ Our EF value, $\sim 5 \times 10^{5}$, is effectively indistinguishable from that obtained by Jung $\left(6 \times 10^{5}\right)$, who also used the Le $\mathrm{Ru}$ approach to calculate the SERS EF from monolayers of benzenethiol on nanostructured surfaces. ${ }^{54}$

\section{CONCLUSIONS}

We demonstrated a method to produce electrically continuous arrays of metal and ITO nanotubes, based on shadow evaporation into the pores of AAO membranes. This technique is attractive because (i) it is simple, (ii) it provides control over the heights and the diameters of the tubes by varying the incident angle of evaporation and the dimensions of the template, respectively, and (iii) it is adaptable to any material that can be vapor-deposited. We have demonstrated, for the first time, the controlled fabrication of ordered arrays of nanotubes of ITO, a particularly important material because it is both conductive and transparent. We also showed that an array of Au nanotubes produced using this method gives a SERS enhancement factor of $\sim 5 \times 10^{5}$ for a monolayer of benzenethiolate. We believe that simple nanofabrication techniques, such as the one described here, that provide large $\left(\sim \mathrm{cm}^{2}\right)$ homogeneous areas of well-defined structure may serve as reliable, effective strategies for producing SERS substrates for applications such as chemical sensors. ${ }^{59}$

The arrays of metal and ITO nanotubes formed using this technique could also serve as high-surface area electrodes - as well as templates for electrochemical growth or conformal deposition of additional materials - for electro-optical applications such as sensors and photovoltaics.

\section{METHODS}

Arrays of Nanotubes. We mounted the AAO membranes (Whatman Anodisc) flush against a rotating plate driven by a battery-powered DC motor ( 30 rpm, Hankscraft). We centered the motor directly over the source and fixed the evaporation incident angle $(\alpha)$ between 0 and $90^{\circ}$ to control the height of the nanotubes. After e-beam evaporating $\sim 150 \mathrm{~nm}$ of metal ( $\mathrm{Au}$ or $\mathrm{Pt}$ ) or ITO ( $\mathrm{ACl}$ Alloys) onto the membranes, we dissolved the alumina by immersing the coated membrane into $1 \mathrm{M} \mathrm{NaOH}(\mathrm{aq})$ for $1 \mathrm{~h}$. This procedure yielded an array of nanotubes supported by an electrically continuous backing of the same material.

Preparation of Samples for SERS Measurement. We shadowevaporated $\mathrm{Au}\left(\alpha=45^{\circ}\right)$ onto an AAO membrane with 200$\mathrm{nm}$-wide pores. We pressed the gold-coated side of the membrane onto a tacky thin film of poly(methyl methacrylate) (PMMA) spin-coated on a $\mathrm{Si} / \mathrm{SiO}_{2}$ wafer and heated the film to $\sim 130{ }^{\circ} \mathrm{C}$ on a hot plate. This film served as a mechanical support for the arrays of tubes, but did not contribute to the SERS signal. After cooling the substrate, we submersed the $\mathrm{Si} / \mathrm{SiO}_{2} / \mathrm{PMMA} / \mathrm{Au} / \mathrm{AAO}$ composite sample in $1 \mathrm{M} \mathrm{NaO}$ $\mathrm{H}(\mathrm{aq})$ for $40 \mathrm{~min}$ to remove the $A A O$, rinsed the sample with deionized water and ethanol, and allowed it to dry in air. We placed the sample in a $1 \mu \mathrm{M}$ solution of benzenethiol (Aldrich, filtered through alumina to remove oxidized species) in ethanol for $8 \mathrm{~h}$. After removing the sample from the solution, we gently rinsed it with ethanol, allowed it to dry in air, and used it within $1 \mathrm{~h}$ of its removal from solution.

Preparation of Reference Samples for SERS Measurements. We sealed a drop $(\sim 0.1 \mathrm{~mL})$ of benzenethiol between a glass slide $(2 \mathrm{in} . \times$ 3 in. $\times \sim 1 \mathrm{~mm})$ and a glass coverslip $(22 \times 22 \times 0.15 \mathrm{~mm})$ using rubber gasket as a spacer (Invitrogen Press and Seal, $0.5 \mathrm{~mm}$ thick).

Acknowledgment. We acknowledge funding from NSF CHE0518055. We used shared facilities supported by the NSF under NSEC (PHY-0646094) and MRSEC (DMR-0213805). E.A.W. thanks the Petroleum Research Fund of the American Chemical Society for a fellowship (PRF no. 43083-AEF). We thank Huiyi Chen and the research group of X. S. Xie for use of their Raman microscope, and Ertugrul Cubukcu and Eric Diebold for helpful discussions.

Supporting Information Available: Further details on image analysis and SERS measurements and Figure S1 (20-200 nm features formed by shadow evaporation). This material is available free of charge via the Internet at http://pubs.acs.org.

\section{REFERENCES AND NOTES}

1. Martin, C. R. Nanomaterials: A Membrane-Based Synthetic Approach. Science 1994, 266, 1961-1966.

2. Lahav, M.; Weiss, E. A.; Xu, Q.; Whitesides, G. M. Core-Shell and Segmented Polymer-Metal Composite Nanostructures. Nano Lett. 2006, 6, 2166-2171.

3. Masuda, H.; Fukuda, K. Ordered Metal Nanohole Arrays Made by a Two-Step Replication of Honeycomb Structures of Anodic Alumina. Science 1995, 268, 1466-1468.

4. Black, C. T.; Guarini, K. W.; Milkove, K. R.; Baker, S. M.; Russell, T. P.; Tuominen, M. T. Integration of SelfAssembled Diblock Copolymers for Semiconductor Capacitor Fabrication. Appl. Phys. Lett. 2001, 79, 409-411.

5. Black, C. T.; Guarini, K. W.; Zhang, Y.; Kim, H.; Benedict, J.; Sikorski, E.; Babich, I. V.; Milkove, K. R. High-Capacity, SelfAssembled Metal-Oxide-Semiconductor Decoupling Capacitors. IEEE Electron Device Lett. 2004, 25, 622-624.

6. Sputtering evaporation typically occurs under a higher pressure (usually a factor of 1000 higher) than does ebeam evaporation. Furthermore, the distance between the source and the target in sputter-coaters is often a factor of two to three smaller than that for e-beam evaporators. These two factors combine to make sputtering a much less directional deposition method than is e-beam. See ref 9.

7. Zhao, S.; Roberge, H.; Yelon, A.; Veres, T. New Application of AAO Template: A Mold for Nanoring and Nanocone Arrays. J. Am. Chem. Soc. 2006, 128, 12352-12353.

8. Lee, W.; Alexe, M.; Nielsch, K.; Goesele, U. Metal Membranes with Hierarchically Organized Nanotube Arrays. Chem. Mater. 2005, 17, 3325-3327.

9. Rossnagel, S. M. Thin Film Deposition with Physical Vapor Deposition and Related Technologies. J. Vac. Sci. Technol., A 2003, 21, S74-S87.

10. Wang, Y.; Zang, K.; Chua, S.; Sander, M. S.; Tripathy, S.; Fonstad, C. G. High-Density Arrays of InGaN Nanorings, Nanodots, and Nanoarrows Fabricated by a TemplateAssisted Approach. J. Phys. Chem. B 2006, 110, 11081-11087.

11. Hua, Z.; Yang, S.; Huang, H.; Lv, L.; Lu, M.; Gu, B.; Du, Y. Metal Nanotubes Prepared by a Sol-Gel Method Followed by a Hydrogen Reduction Procedure. Nanotechnology 2006, 17, 5106-5110.

12. Martin, C. R. Template Synthesis of Electronically Conductive Polymer Nanostructures. Acc. Chem. Res. 1995, 28, 61-68. 
13. Sander, M. S.; Tan, L.-S. Nanoparticle Arrays on Surfaces Fabricated Using Anodic Alumina Films as Templates. Adv. Funct. Mater. 2003, 13, 393-397.

14. Long, J. W.; Dunn, B.; Rolison, D. R.; White, H. S. ThreeDimensional Battery Architectures. Chem. Rev. 2004, 104, 4463-4492.

15. Wei, Q.; Zheng, H.; Huang, Y. Direct Patterning ITO Transparent Conductive Coatings. Sol. Energy Mater. Sol. Cells 2001, 68, 383-390.

16. Cho, S. I.; Choi, D. H.; Kim, S.-H.; Lee, S. B. Electrochemical Synthesis and Fast Electrochromics of Poly(3,4Ethylenedioxythiophene) Nanotubes in Flexible Substrate. Chem. Mater. 2005, 17, 4564-4566.

17. Cho, S. I.; Kwon, W. J.; Choi, S.-J.; Kim, P.; Park, S.-A.; Kim, J.; Son, S. J.; Xiao, R.; Kim, S.-H.; Lee, S. B. Nanotube-Based Ultrafast Electrochromic Display. Adv. Mater. 2005, 17, $171-175$.

18. Aoki, Y.; Huang, J.; Kunitake, T. Electro-Conductive Nanotubular Sheet of Indium Tin Oxide as Fabricated from the Cellulose Template. J. Mater. Chem. 2006, 16, 292-297.

19. Wan, Q.; Song, Z. T.; Feng, S. L.; Wang, T. H. SingleCrystalline Tin-Doped Indium Oxide Whiskers: Synthesis and Characterization. Appl. Phys. Lett. 2004, 85, 4759-4761.

20. Limmer, S. J.; Cruz, S. V.; Cao, G. Z. Films and Nanorods of Transparent Conducting Oxide ITO by a Citric Acid Sol Route. Appl. Phys. A: Mater. Sci. Process. 2004, 79, 421-424.

21. Wan, Q.; Feng, P.; Wang, T. H. Vertically Aligned Tin-Doped Indium Oxide Nanowire Arrays. Epitaxial Growth and Electron Field Emission Properties. Appl. Phys. Lett. 2006, 89, 123102/1-3.

22. Cheng, Z.-X.; Dong, X.-B.; Pan, Q.-Y.; Zhang, J.-C.; Dong, X.W. Preparation and Characterization of $\ln _{2} \mathrm{O}_{3}$ Nanorods. Mater. Lett. 2006, 60, 3137-3140.

23. Xue, X. Y.; Chen, Y. J.; Liu, Y. G.; Shi, S. L.; Wang, Y. G.Wang, T. H. Synthesis and Ethanol Sensing Properties of Indium-Doped Tin Oxide Nanowires. Appl. Phys. Lett. 2006, 88, 201907/1-3.

24. Jang, H. S.; Kim, D.-H.; Lee, H.-R.; Lee, S.-Y. Field Emission from Cone-Like Single Crystalline Indium Tin Oxide Nanorods. Mater. Lett. 2005, 59, 1526-1529.

25. Yu, D.; Wang, D.; Yu, W.; Qian, Y. Synthesis of Ito Nanowires and Nanorods with Corundum Structure by a CoPrecipitation-Anneal Method. Mater. Lett. 2003, 58, 84-87.

26. Orlandi, M. O.; Aguiar, R.; Lanfredi, A. J. C.; Longo, E.; Varela, J. A.; Leite, E. R. Tin-Doped Indium Oxide Nanobelts Grown by Carbothermal Reduction Method. Appl. Phys. A: Mater. Sci. Process. 2004, 80, 23-25.

27. Tian, M.; Xu, S.; Wang, J.; Kumar, N.; Wertz, E.; Li, Q.; Campbell, P. M.; Chan, M. H. W.; Mallouk, T. E. Penetrating the Oxide Barrier in Situ and Separating Freestanding Porous Anodic Alumina Films in One Step. Nano Lett. 2005, 5, 697-703.

28. Lee, W.; Ji, R.; Goesele, U.; Nielsch, K. Fast Fabrication of Long-Range Ordered Porous Alumina Membranes by Hard Anodization. Nat. Mater. 2006, 5, 741-747.

29. Li, A. P.; Muller, F.; Birner, A.; Nielsch, K.; Gosele, U. Hexagonal Pore Arrays with a 50-420 nm Interpore Distance Formed by Self-Organization in Anodic Alumina. J. Appl. Phys. 1998, 84, 6023-6026.

30. Ono, S.; Masuko, N. Evaluation of Pore Diameter of Anodic Porous Films Formed on Aluminum. Surf. Coat. Technol. 2003, 169-170, 139-142.

31. Furneaux, R. C.; Rigby, W. R.; Davidson, A. P. The Formation of Controlled-Porosity Membranes from Anodically Oxidized Aluminum. Nature 1989, 337, 147-149.

32. Xie, Y. Photoelectrochemical Reactivity of a Hybrid Electrode Composed of Polyoxophosphotungstate Encapsulated in Titania Nanotubes. Adv. Funct. Mater 2006, 16, 1823-1831.

33. Paulose, M.; Shankar, K.; Yoriya, S.; Prakasam, H. E.; Varghese, O. K.; Mor, G. K.; Latempa, T. A.; Fitzgerald, A.; Grimes, C. A. Anodic Growth of Highly Ordered $\mathrm{TiO}_{2}$ Nanotube Arrays to $134 \mu \mathrm{m}$ in Length. J. Phys. Chem. $B$ 2006, 110, 16179-16184.
34. Mor, G. K.; Varghese, O. K.; Paulose, M.; Shankar, K.; Grimes, C. A. A Review on Highly Ordered, Vertically Oriented $\mathrm{TiO}_{2}$ Nanotube Arrays: Fabrication, Material Properties, and Solar Energy Applications. Sol. Energy Mater. Sol. Cells 2006, 90, 2011-2075.

35. Prakasam, H. E.; Shankar, K.; Paulose, M.; Varghese, O. K.; Grimes, C. A. A New Benchmark for $\mathrm{TiO}_{2}$ Nanotube Array Growth by Anodization. J. Phys. Chem. C 2007, 111, 7235-7241.

36. Mor, G. K.; Varghese, O. K.; Paulose, M.; Grimes, C. A. Transparent Highly Ordered $\mathrm{TiO}_{2}$ Nanotube Arrays Via Anodization of Titanium Thin Films. Adv. Funct. Mater. 2005, 15, 1291-1296.

37. Macak, J. M.; Tsuchiya, H.; Taveira, L.; Aldabergerova, S.; Schmuki, P. Smooth Anodic $\mathrm{TiO}_{2}$ Nanotubes. Angew. Chem. 2005, 44, 7463-7465.

38. Wang, J.; Li, Y. Rational Synthesis of Metal Nanotubes and Nanowires from Lamellar Structures. Adv. Mater. 2003, 15 , 445-447.

39. Kijima, T.; Yoshimura, T.; Uota, M.; Ikeda, T.; Fujikawa, D.; Mouri, S.; Uoyama, S. Noble-Metal Nanotubes (Pt, Pd, Ag) from Lyotropic Mixed-Surfactant Liquid-Crystal Templates. Angew. Chem. 2004, 43, 228-232.

40. Sun, Y.; Xia, Y. Multiple-Walled Nanotubes Made of Metals. Adv. Mater. 2004, 16, 264-268.

41. Cao, H.; Wang, L.; Qiu, Y.; Wu, Q.; Wang, G.; Zhang, L.; Liu, X. Generation and Growth Mechanism of Metal (Fe, Co, Ni) Nanotube Arrays. ChemPhysChem 2006, 7, 1500-1504.

42. Ku, J.-R.; Vidu, R.; Talroze, R.; Stroeve, P. Fabrication of Nanocables by Electrochemical Deposition inside Metal Nanotubes. J. Am. Chem. Soc. 2004, 126, 15022-15023.

43. Lee, W.; Moyen, E.; Wulfhekel, W.; Leycuras, A.; Nielsch, K.; Goesele, U.; Hanbuecken, M. Vertical Nanopatterning of 6 h-Sic(0001) Surfaces Using Gold-Metal Nanotube Membrane Lithography. Appl. Phys. A: Mater. Sci. Process. 2006, 83, 361-363.

44. Mor Gopal, K.; Shankar, K.; Paulose, M.; Varghese Oomman, K.; Grimes Craig, A. Use of Highly-Ordered $\mathrm{TiO}_{2}$ Nanotube Arrays in Dye-Sensitized Solar Cells. Nano Lett. 2006, 6, 215-218.

45. Wang, S.; Yu, G. J.; Gong, J. L.; Li, Q. T.; Xu, H. J.; Zhu, D. Z.; Zhu, Z. Y. Large-Area Fabrication of Periodic Fe Nanorings with Controllable Aspect Ratios in Porous Alumina Templates. Nanotechnology 2006, 17, 1594-1598.

46. Wang, S.; Yu, G. J.; Gong, J. L.; Zhu, D. Z.; Xia, H. H. LargeArea Uniform Nanodot Arrays Embedded in Porous Anodic Alumina. Nanotechnology 2007, 18, 015303/015301-015303/015304.

47. Hobbs, K. L.; Larson, P. R.; Lian, G. D.; Keay, J. C.; Johnson, M. B. Fabrication of Nanoring Arrays by Sputter ReDeposition Using Porous Alumina Templates. Nano Lett. 2004, 4, 167-171.

48. Kobayashi, H.; Ishida, T.; Nakamura, K.; Nakato, Y.; Tsubomura, H. Properties of Indium Tin Oxide Films Prepared by the Electron Beam Evaporation Method in Relation to Characteristics of Indium Tin Oxide/Silicon Oxide/Silicon Junction Solar Cells. J. Appl. Phys. 1992, 72, 5288-5293.

49. Hammiche, A.; Webb, R. P.; Wilson, I. H. A Scanning Tunnelling Microscopy Study of Thin Gold Films Evaporated on Silicon. Vacuum 1994, 45, 569-573.

50. Sze, S. M.; Ng, K. K. Physics of Semiconductor Devices, 3rd ed.; Wiley-Interscience: Hoboken, NJ, 2007; pp 31-32.

51. George, J.; Menon, C. S. Electrical and Optical Properties of Electron Beam Evaporated ITO Thin Films. Surf. Coat. Technol. 2000, 132, 45-48.

52. Kneipp, K.; Wang, Y.; Kneipp, H.; Perelman, L. T.; Itzkan, I.; Dasari, R. R.; Feld, M. S. Single Molecule Detection Using Surface-Enhanced Raman Scattering (SERS). Phys. Rev. Lett. 1997, 78, 1667-1670.

53. Nie, S.; Emory, S. R. Probing Single Molecules and Single Nanoparticles by Surface-Enhanced Raman Scattering. Science 1997, 275, 1102-1106. 

Facile Fabrication of Large Area Nanostructures for Efficient Surface-Enhanced Raman Scattering. J. Mater. Chem. 2006, 16, 3145-3149.

55. Dieringer, J. A.; McFarland, A. D.; Shah, N. C.; Stuart, D. A.; Whitney, A. V.; Yonzon, C. R.; Young, M. A.; Zhang, X.; Van Duyne, R. P. Surface Enhanced Raman Spectroscopy: New Materials, Concepts, Characterization Tools, and Applications. Faraday Discuss. 2006, 132, 9-26.

56. Cintra, S.; Abdelsalam, M. E.; Bartlett, P. N.; Baumberg, J. J.; Kelf, T. A.; Sugawara, Y.; Russell, A. E. Sculpted Substrates for Sers. Faraday Discuss. 2006, 132, 191-199.

57. Felidj, N.; Aubard, J.; Levi, G.; Krenn, J. R.; Salerno, M.; Schider, G.; Lamprecht, B.; Leitner, A.; Aussenegg, F. R. Controlling the Optical Response of Regular Arrays of Gold Particles for Surface-Enhanced Raman Scattering. Phys. Rev. B: Condens. Matter Mater. Phys 2002, 65, 075419/1-9.

58. Cai, W. B.; Ren, B.; Li, X. Q.; She, C. X.; Liu, F. M.; Ca, X. W.; Tian, Z. Q. Investigation of Surface-Enhanced Raman Scattering from Platinum Electrodes Using a Confocal Raman Microscope: Dependence of Surface Roughening Pretreatment. Surf. Sci. 1998, 406, 9-22.

59. Natan, M. J. Concluding Remarks Surface Enhanced Raman Scattering. Faraday Discuss. 2006, 132, 321-328.

60. Perney, N. M. B.; Garcia de Abajo, F. J.; Baumberg, J. J.; Tang, A.; Netti, M. C.; Charlton, M. D. B.; Zoorob, M. E. Tuning Localized Plasmon Cavities for Optimized SurfaceEnhanced Raman Scattering. Phys. Rev. B: Condens. Matter Mater. Phys 2007, 76, 035426/1-5.

61. Baumberg, J. J.; Kelf, T. A.; Sugawara, Y.; Cintra, S.; Abdelsalam, M. E.; Bartlett, P. N.; Russell, A. E. AngleResolved Surface-Enhanced Raman Scattering on Metallic Nanostructured Plasmonic Crystals. Nano Lett. 2005, 5, 2262-2267.

62. Varsányi, G.; Kovner, M. A.; Láng, L. Assignments for Vibrational Spectra of 700 Benzene Derivatives; John Wiley \& Sons: New York, 1974; p 668.

63. Le Ru, E. C.; Blackie, E.; Meyer, M.; Etchegoin, P. G. Surface Enhanced Raman Scattering Enhancement Factors: A Comprehensive Study. J. Phys. Chem. C 2007, 111, 1379413803.

64. The so-called "total SERS substrate EF" - the value obtained when the entire area of the Au substrate is considered in the $N_{\text {surf }}$ calculation (i.e., $F_{\text {surf }}=1$ ) - is therefore approximately a factor of 5 lower than the EF.

65. Wan, L.-J.; Terashima, M.; Noda, H.; Osawa, M. Molecular Orientation and Ordered Structure of Benzenethiol Adsorbed on Gold(111). J. Phys. Chem. B 2000, 104, 35633569. 